\title{
Underutilization of Diabetes Education. Experience in an Urban Teaching Hospital in The Bronx
}

Edwin A Torres ${ }^{1}$, Akankasha Tiwari ${ }^{2}$, Sharon Movsas ${ }^{2}$, Iris Carrasquillo ${ }^{3}$ and Joel Zonszein ${ }^{2 *}$

${ }^{1}$ Binghamton University-Decker School of Nursing, NY, USA

${ }^{2}$ Montefiore Medical Center, The University Hospital of the Albert Einstein College of Medicine, Department of Medicine, Division of Endocrinology, NY, USA

${ }^{3}$ Montefiore Medical Center, The University Hospital of the Albert Einstein College of Medicine, Department of Nursing, NY, USA

\begin{abstract}
Objective: Patient education is a fundamental component of diabetes management. Few studies have evaluated the "real life" frequency and impact of diabetes education. This study, conducted in a large teaching hospital in Bronx, New York, focused on a variety of diabetes educational programs in order to assess their utilization by patients and their individual impact.

Research design and methods: Face-to-face interviews were conducted with a convenience sample of patients with type 2 diabetes, both inpatient and outpatient, by registered nurses or physicians. Information about demographics, type of education provided, and patient knowledge of hemoglobin A1C (A1C), lipids, and blood pressure co1ntrol was obtained using a questionnaire.

Results: Seventy-four adult patients were interviewed between January 2013 and December 2013. Education was provided to 38\% of the sample with a similar distribution among inpatients $(38 \%)$ and outpatients (33\%). The majority received one-to-one "unstructured education." Education had no impact on knowledge and/or clinical parameters. No differences were found among the $\mathrm{A} 1 \mathrm{C}$ values between those that received and did not receive education (A1C $8.6 \%$ vs $8.3 \%$ ), or among those hospitalized (A1C $8 \%$ ) versus treated as outpatients (A1C 8.4\%). Similarly, LDL-cholesterol did not differ between those who received and did not receive education (85 mg/dl vs $89 \mathrm{mg} / \mathrm{dl}$ respectively).
\end{abstract}

*Corresponding author: Joel Zonszein, Montefiore Medical Center, Department of Medicine, Division of Endocrinology and Metabolism, 1880 Morris Park Avenue, Bronx, New York, 10461, USA, Tel: +1 3474982445; Fax: +1 7182398285; E-mail: JZonszei@montefiore.org

Citation: Torres EA, Tiwari A, Movsas S, Carrasquillo I, Zonszein J (2015) Underutilization of Diabetes Education. Experience in an Urban Teaching Hospital in The Bronx. J Diabetes Metab Disord 2: 005.

Received: February 11, 2015; Accepted: April 09, 2015; Published: April 23, 2015

Copyright: () 2015 Torres EA, et al., This is an open-access article distributed under the terms of the Creative Commons Attribution License, which permits unrestricted use, distribution, and reproduction in any medium, provided the original author and source are credited.
Conclusion: Despite ample availability of educational programs in a large urban teaching medical center, most patients did not receive education, and when provided, it was unstructured. We identified and discussed potential barriers that may have contributed to the underutilization and quality of education. However, there remains an opportunity to increase the use of and improve the quality of diabetes education.

\section{Introduction}

Type 2 Diabetes Mellitus (T2DM) has reached epidemic proportions worldwide. The prevalence is currently estimated at 345 million and is expected to reach 500 million by 2030 [1]. The Bronx in New York City is a 'hot spot' for diabetes with a prevalence of $14.6 \%$ among adults, one of the highest in the country. While the Bronx is one of five boroughs in New York City, its population is larger than all but five US cities. The Bronx is made up of a vibrant and younger population of diverse racial/ethnic backgrounds that is disproportionately afflicted with a high rate of diabetes and its complications [2].

Patient education is essential in the management of chronic diseases such as diabetes and has been found to be cost effective and improve clinical outcomes [3-4]. Diabetes is a demanding and complex disease in which the bulk of the management is performed by the patient. Education provides knowledge, skills, and behavioral strategies needed to make numerous daily decisions and improve medication adherence particularly when polypharmacy is used [5-6]. Educational programs consistent with the National Standards for Diabetes Self-Management Education (DSME) and support are recommended at diagnosis for all patients as a standard of practice [7-8]. However, in large urban medical institutions different programs are often needed to serve the diverse patient population [9-10].

Yet, even with strong evidence of better outcomes and widespread reimbursement, education remains an underutilized resource. Less than half of the individuals diagnosed with diabetes receive diabetes education, with even lower percentages found in large cities $[11,12]$. A recent study in the U.S. using claims data found that less than $7 \%$ of adults with diabetes who had medical insurance participated in DSME programs [13]. While many studies have established the importance of diabetes education, few examine the quality and extent to which it is utilized by patients in "real-life" clinical settings where such programs are available. In an effort to address this gap, we conducted a survey in a large urban teaching hospital in Bronx, New York where several diabetes educational programs are available to meet the needs of a diverse and high-risk population for diabetes and its complications.

\section{Design and Methods}

In this ex-post-facto study design, 74 adult patients with diabetes were interviewed during hospitalization or at outpatient clinics. The hospitalized patients were admitted for various non-diabetes related complications and co morbidities. Patients with acute dysglycemic complications such as hypoglycemia, diabetic ketoacidosis, or hyperosmolar hyperglycemic state were excluded from this study.

Data was obtained between January 2013 and December 2013. A one-on-one interview was conducted privately in English or Spanish 
Citation: Torres EA, Tiwari A, Movsas S, Carrasquillo I, Zonszein J (2015) Underutilization of Diabetes Education. Experience in an Urban Teaching Hospital in The Bronx. J Diabetes Metab Disord 2: 005.

\begin{tabular}{|c|c|}
\hline Questions & Answers \\
\hline Have you ever been in a diabetes education program or diabetes class? & Yes, No, Not sure/Don't know \\
\hline When in the disease was it provided? & At diagnosis, $<5$ years duration, $5-10$ years, $>10$ years \\
\hline What type? & Individual, Group classes, Internet, Telephonic, Other \\
\hline Do you know who provided the education? & MD, RD, RN, Other, Not sure/Don't know \\
\hline What was the duration of the education? & Less than hr, $1 \mathrm{hr}, 2 \mathrm{hrs}, 5 \mathrm{hrs}, 10 \mathrm{hrs}$. Not sure/Don't know \\
\hline Have you ever attended diabetes support groups? & A) Yes \\
\hline \multicolumn{2}{|c|}{ Disease Knowledge } \\
\hline 1. What type of Diabetes do you have? & T1DM, T2DM, Not sure/Don't know \\
\hline 2. How long do you have diabetes? & $<1,1-5,5-10,>10$ (years), Not sure/Don't know \\
\hline 3. What is an $\mathrm{A} 1 \mathrm{C}$ ? & Knows, Not sure, Don't know \\
\hline 4. Do you know what your last value was? & $<7 \%, 7-9 \%,>9 \%$, Not sure/Don't know \\
\hline 6. Do you have hypertension (high blood pressure)? & Yes, No, Not sure/Don't know \\
\hline 7. If yes is it well controlled? & Yes, No, Not sure/Don't know \\
\hline 8. Do you take medication for hypertension? & Yes, No, Not sure/Don't know \\
\hline 9. Do you have high cholesterol? & Yes, No, Not sure/Don't know \\
\hline 10. If yes, is it well controlled? & Yes, No, Not sure/Don't know \\
\hline 11. Do you take medication for high cholesterol? & Yes, No, Not sure/Don't know \\
\hline
\end{tabular}

Table 1: Education questionnaire sample summary.

according to the patient's preference, either by Hispanic or non-Hispanic nurses or physicians. The information was obtained using a survey, summarized in table 1 , that was developed, pilot tested, and revised for this project but not validated. Montefiore Medical Center (MMC) and the Albert Einstein College of Medicine Institutional Review Board approved the final protocol and questionnaire. Each participant was asked for an oral informed consent and completed a brief survey. No gift or honorarium was provided.

Demographic and socioeconomic variables were constructed based on answers by the surveyed patients. Patients were categorized as non-Hispanic White, non-Hispanic Black, Hispanic/Latino, Asian, or Other. The patients' marital status was grouped as married, living with partner, divorced, widowed, separated, never married, or single. Information regarding patient education level, median household income, and type of medical health insurance during the prior year was also obtained. Patients were asked what type of diabetes they had and if they had received diabetes education. If they did, there was further inquiry about the type of education and when over the course of their disease it was provided. Responses of "don't know" and "refused" were coded as missing.

Knowledge was assessed based on answers about what a hemoglobin $\mathrm{A} 1 \mathrm{C}$ (A1C) is, the A1C goal, the patient's own result, and when it was last done. These questions were also asked about cholesterol and blood pressure. Following the interview, electronic medical records were used to extract data regarding the actual $\mathrm{A} 1 \mathrm{C}$ and lipid values done within the last three months in order to correlate education with biochemical markers. Data from the questionnaires was coded and extracted for statistical quantitative analysis using the SPSS-version 21 programs. An independent sample t-test was calculated comparing the means scores of both, outpatient and inpatient patients. No statistical significant was found among education variables $(\mathrm{t}(72)=0.770, \mathrm{p}>05)$. Our dependent variables, education knowledge and metabolic biomarkers were not significant among the two groups.

\section{Results}

The study population was representative of the Bronx (Table 2), a predominantly poor area of New York City with a diverse population and high prevalence of obesity and T2DM with its complications [14]. Amongst the seventy-four patients interviewed, 9.5\% had type 1 Diabetes Mellitus (T1DM), 64\% had T2DM, and 26\% did not know their type of diabetes based on self-identification. The mean age was 60.3 years and a high proportion were women $(64.9 \%)$. The majority were Hispanic/Latinos (54.1\%), of whom $24.3 \%$ were non-Hispanic Black and $40 \%$ were foreign-born. There was a high rate of poverty (13.9\%). Thirty-four percent of individuals had a yearly household income of less than $\$ 30,000.00$ US Dollars (USD) and $20 \%$ had an income greater than $\$ 50,000.00$ USD. Medical insurance coverage was available to the majority (89.2\%), with $56.8 \%$ having Medicare or Medicaid. Only $17.6 \%$ had less than an eighth grade education and $64 \%$ attended high school or beyond. Disability was present in $16 \%$ and $9.5 \%$ were unemployed.

Diabetes education was reported by $38 \%$ with a similar distribution among inpatients (38\%) and outpatients (33\%) (Table 3). Of those who received education, the majority ( $18 \%$ - not shown) had one-to-one, non-structured education given within the first five years of diagnosis. None attended the 10-hour DSME group workshops. There was no impact of education on knowledge or clinical outcomes. While $52 \%$ were familiar with the term A1C, only $26 \%$ understood what it represents and $23 \%$ knew the approximate date when it was last checked. The actual A1C values indicated no significant differences between patients who did (8.7\%) and did not (8.3\%) receive education. Similarly, there was no difference in A1C between inpatients $(8.0 \%)$ versus outpatients $(8.4 \%)$. Thirty-seven percent had an $\mathrm{A} 1 \mathrm{C}>7 \%$ with no correlation to diabetes education. A non-significant difference $(p>0.05)$ was also found in lipid control as 


\begin{tabular}{|c|c|}
\hline & $\mathrm{N}(\%)$ \\
\hline & $74(100 \%)$ \\
\hline \multicolumn{2}{|c|}{ Characteristics* } \\
\hline Mean age in years (range) & $60(30-89)$ \\
\hline Gender (female) & $48(65)$ \\
\hline \multicolumn{2}{|c|}{ Race/ethnicity } \\
\hline Non-Hispanic Black & $18(24.3)$ \\
\hline Hispanic/Latino & $40(54.1)$ \\
\hline Non-Hispanic White & $9(12.2)$ \\
\hline Asian & $1(1.4)$ \\
\hline Born in the U.S & $44(60)$ \\
\hline Puerto Rico & $11(15)$ \\
\hline \multicolumn{2}{|c|}{ Diabetes Type } \\
\hline Type 1 & $7(10)$ \\
\hline Type 2 & $47(64)$ \\
\hline Don't know & $18(26)$ \\
\hline Outpatient & $37(50)$ \\
\hline Inpatient & $37(50)$ \\
\hline Health insurance coverage & $66(89)$ \\
\hline Medicare/Medicaid & $42(57)$ \\
\hline \multicolumn{2}{|c|}{ Education } \\
\hline Less than $12^{\text {th }}$ grade education & $48(65)$ \\
\hline High School / Technical School & $10(14)$ \\
\hline Completed College & $15(20)$ \\
\hline \multicolumn{2}{|c|}{ Employment } \\
\hline Working full time & $14(19.9)$ \\
\hline Working part time & $7(9.5)$ \\
\hline Unemployed & $14(19)$ \\
\hline Disabled & $12(16.2)$ \\
\hline Married & $37(50)$ \\
\hline \multicolumn{2}{|c|}{ Yearly household income } \\
\hline Less than $\$ 30,000$ & $25(34)$ \\
\hline$\$ 30,000$ to $\$ 50,000$ & $12(16)$ \\
\hline More than $\$ 50,0000$ & $15(20)$ \\
\hline
\end{tabular}

Table 2: Demographics.

* Total \% does not include others

** Diabetes type was self-reported by each subject

shown in table 3.When social determinants and medical health insurance were analyzed, no correlation to education was found.

\begin{tabular}{|l|c|c|}
\hline \multicolumn{1}{|c|}{ Variable } & $\begin{array}{l}\text { Received Unstructured } \\
\text { Education* }^{*}\end{array}$ & No Education* \\
\hline Unstructured diabetes Education (\%) & 38 & 62 \\
\hline What is an A1C? (\%) & 38 & 62 \\
\hline A1C value (mean \%) & 8.6 & 8.3 \\
\hline Total-Cholesterol (mean mg/dl) & 166 & 173 \\
\hline Triglycerides (mean mg/dl) & 152 & 167 \\
\hline LDL-Cholesterol (mean mg/dl) & 85 & 89 \\
\hline HDL-Cholesterol (mean mg/dl) & 50 & 54 \\
\hline
\end{tabular}

Table 3: Education, Knowledge and Biochemical Parameters. ${ }^{*} p$ value $>0.05$

\section{Discussion}

Our results derived from a "real world" urban clinical setting confirm that diabetes education remains underutilized in a large integrated medical system in the Bronx where programs for education have been widely available. When education was provided, it had little or no impact on knowledge or clinical outcomes, suggesting that it was not effective. Poor participation in educational programs is not unique to the Bronx and occurs across the country, even in patients with medical insurance [13]. In this study, we present several barriers and challenges, based on our observations at MMC, which appear to play a role in the low rate and poor quality. Education and educational programs vary greatly among institutions in urban hospitals, but may share similar barriers.

\section{Montefiore diabetes programs in the Bronx}

In the Bronx, diabetes is a major problem afflicting a diverse and young population with high rates of complications and hospitalizations [15]. Montefiore Medical Center (MMC), the University Hospital of the Albert Einstein College of Medicine is the largest integrated health care delivery system in the Bronx and provides care to more than half of the Bronx population. In response to the community, several diabetes initiatives were created placing education at their core. While these educational programs are integrated, they function as independent entities. The cornerstone program is the MMC-Clinical Diabetes Center that consists of teams of Registered Nurses (RNs) and Registered Dietitians (RDs) working with endocrinologists, all Certified Diabetes Educators (CDEs). It has an inpatient and outpatient component. The goal of the in-hospital program is to help patients navigate their hospital stay with better glycemic control and provide basic need-to-know education. Another major objective is helping transition to the outpatient setting. In-patients are invited to participate in one of several educational programs that are offered at various locations and times to meet patients' needs after their hospitalization.

Each education program is tailored specifically to the patients' type of diabetes and population served. Adult individuals with T1DM are referred for one-to-one education for intensification of glycemic control. Pregnant women participate in specific programs for diabetes in pregnancy with emphasis in family care and prevention. Ideally, adults with T2DM attend a 10-hour DSME program recognized by the American Diabetes Association (ADA), the most structured and comprehensive of the programs. A consultation letter documenting patient participation, diagnostic and therapeutic recommendations is sent to the referring primary care provider. Other less-structured programs are also available where education is typically provided on a one-to-one basis, particularly to those patients with very poor glycemic control and poor adherence to the medical regimens $[16,17]$.

Because of the ethnic diversity of patient populations, social issues, and difficulty with access to healthcare in the Bronx, several other initiatives exist. A Diabetes Disease Management Program (DDMP) that evolved as an important component for diabetes care used by the MMC Accountable Care Organization (ACO) was created [18]. It exists in many of the satellite primary care clinic sites and serves as a guide for RNs, RDs, pharmacists, the majority CDE's that work closely with physicians and other health care providers. Patients early in the course of disease or those with better glycemic control are usually referred to the 10 -hour DSME program at the diabetes center 
[19]. For patients with poor glycemic control, education is typically provided one-to-one but the DDMP also offers abbreviated, less structured, unrecognized programs. Several other group and one-to-one educational programs are also in place for the diabetes clinic and faculty practice. Other programs exist that are provided by non-diabetes specialists, or health care educators referred as "diabetes champions." Thus, MMC provides a wide range of programs to meet the varied needs of its large Bronx community. It would be reasonable to expect that more patients would be enrolled in one of these programs. Our study, however, shows that these programs are underutilized and the few who were exposed to education, received an unstructured, "reactive" one-to-one encounter that took place only once or in two sessions.

\section{Barriers encountered in the dissemination of diabetes education}

In spite of the many programs available only $38 \%$ reported receiving education. Social status, race/ethnicity, educational level, or insurance coverage did not appear to be determinants for the lack of education. The major barriers appeared to be in the areas of system management and provider issues that may affect the dissemination or access to education. For instance, PCP's are unaware of the existing programs or which programs are best suited for their patients. The referral process is often cumbersome and involves familiarity with details of coverage policies, eligibility requirements, content of the referral, and allowed time. This process often requires back and forth faxes, telephone calls and navigation through the complicated Electronic Medical (EMR) system. A more intuitive, and user-friendly referral process is needed such as an EMR system that has an education section with a menu of choices for easy referral systems that include seamless instructions with the exact venue, date, and duration of the education programs.

Personal and social issues often may interfere with attendance, especially among the underserved populations. This is further compounded by the demoralizing experiences at the clinics which often involve navigating unfriendly hospital systems, coping with long wait times, fragmented care, and inadequate coordination of services. Addressing the various socio-economic patient barriers as well as institutional barriers can be complex and covers a wide scope of issues. It requires collaboration at various social levels, by the individual, family, and community at large. Integrated medical care, proper use of resources and effective electronic medical systems needs to be used to improve the quality of care, streamline referral processes, and increase exposure to quality education.

\section{Why diabetes education may not be effective}

An important finding of this study showed no differences in clinical outcomes among those who received education suggesting that it was not effective. A wide range of variability exists amongst programs, at one end of the spectrum are carefully organized, well-structured programs such as the nationally recognized ones that are built upon evidence-based standards of education shown to produce positive outcomes. These programs consist of a solid structure, qualified instructors, a comprehensive curriculum, participant and program evaluation outcomes, and patient follow-up. At the other end of the spectrum are education programs or encounters without adequate structure, content, or follow-up. They tend to be "reactive" or "casual" such as receiving a single on-demand session by someone not specialized in diabetes or answering questions as part of a visit or discussing dietary issues. In our survey, of those who received education, the majority received one or two sessions of unstructured education and none attended the structured 10-hour DSME program. There is no single best approach to education, and some education is better that no education, but well-structured programs perform better. Specially designed programs may be needed for specific populations. For instance, programs using navigator, "promotores de la salud" or community health workers, dedicated to helping individuals and families navigate through the complexities of the current medical systems serve as "bridges" between community members and health care services and can be effective. The need for these programs is important particularly in areas with diverse populations, different languages, and varying levels of education.

Barriers to creating and maintaining effective programming need to be addressed. Effective programs can be expensive due to the ongoing, rigorous efforts requiring additional staff as well as the actual cost of recognition. However, these programs have been shown to be cost-effective and deliver positive outcomes. Reimbursement is no longer a major barrier as accredited programs are now reimbursable by Medicare, Medicaid, and many commercial insurances. The numerous requirements for recognition are a barrier. They need to have a sponsor provider, or organization enrolled in Medicare with a National identifier provider number, and patients need to have a referral order from their health care provider. Hopefully, these barriers will be ameliorated with health care reform where education is a priority and widely encouraged in Patient centered medical homes and ACO's. Using bundled payments rather than troublesome fee for services, and having integrated approaches with emphasis in disease prevention, should improve the health status of patients with chronic diseases such as diabetes.

The timing when education is provided after the diagnosis is established, is important, the earlier the better. This study found that the patients who received education, it was provided within the first five years of diagnosis. It is not uncommon for providers to refer patients for education late in their disease. Some providers may not be aware of the importance of early education, particularly in those individuals "doing well." Patients are often first sent for education only after failure to control glycaemia or once non-adherence to medications occurs, or when complications already exist. The average disease duration of patients referred to the MMC-DSME program is seven years from the time of diagnosis, supporting the concept that PCPs need to refer patients earlier. A prescription for education should be given to patients with diabetes upon diagnosis with a strong message conveying the importance of early education. Early interventions even before the diagnosis is established are beneficial as demonstrated by the Diabetes Prevention Program (DPP), a clinical research study that showed positive outcomes, and has now been translated to clinical practice [20]. Currently, this program is covered by several health care insurances, in individuals at high risk to develop diabetes. At MMC, we have a community-based program known as Diabetes Education and Prevention with lifestyle Intervention offered at the YMCA and at two more at MMC sites. Programs for early intervention are being utilized more often with positive outcomes [21].

The frequency and duration or "dose" of education is vital; the higher the dose, the better the outcome. A one-time cluster intervention does not have the same impact that higher frequency education may have [22]. This survey found that the majority of individuals received education early with a low dose, with only one or 
Citation: Torres EA, Tiwari A, Movsas S, Carrasquillo I, Zonszein J (2015) Underutilization of Diabetes Education. Experience in an Urban Teaching Hospital in The Bronx. J Diabetes Metab Disord 2: 005.

two encounters. Thus, the type of education as well as the dose of education is vital for positive outcomes. It was expected that hospitalized patients with more co morbidities would have less education and a worse metabolic profile when compared to the outpatients interviewed, but this was not the case in this small study. Hospitalized patients need to receive basic education. More importantly, they need to be referred to more comprehensive programs after their hospitalization as part of the transition of care.

Limitations of this study include a small sample size and recruitment of patients from a single institution. Outcomes may vary among the different clinic sites in one institution and certainly among different sites in parts of the country. The questionnaire was not validated, but it was tailored and modified to the population surveyed. The study however was successful in obtaining a real life cross-sectional estimate of frequency and quality of education provided in a large urban integrated medical center in the Bronx, New York.

In summary, diabetes education, an essential component of diabetes management, is underutilized in "real life" clinical settings and when provided has little impact on patient outcomes. We discuss the various barriers encountered in providing education to patients with diabetes. The implications suggest that educational programs need to be better disseminated and allow for easier patient access. Health care providers need to be aware of the programs in existence, and know how to refer patients early in their disease even when managed well and before complications develop. System problems remain a major barrier but should improve by creating seamless and easier electronic referrals. Education is more effective when provided by qualified health care providers in the "proper dose" and tailored to individual patients. Evaluation of program outcomes is essential in order to assess outcomes and/or modify the program curriculum. Education remains underutilized and there is an opportunity for improvement by optimizing the frequency and quality. Early interventions need to be integrated with high quality and well-structured programs. While our findings are limited by a small sample size, and done in specific populations, larger studies need to be designed to assess the quality, frequency, and impact of diabetes education.

\section{References}

1. van Dieren S, Beulens JW, van der Schouw YT, Grobbee DE, Neal B (2010) The global burden of diabetes and its complications: An emerging pandemic. Eur J Cardiovasc Prev Rehabil 17: 3-8.

2. Hulkower RD, Pollack RM, Zonszein J (2014) Understanding hypoglycemia in hospitalized patients. Diabetes Manag (Lond) 4: 165-176.

3. Norris SL, Engelgau MM, Narayan KM (2001) Effectiveness of self-management training in type 2 diabetes: a systematic review of randomized controlled trials. Diabetes care 24: 561-587.

4. Haas L, Maryniuk M, Beck J, Cox CE, Duker P, et al. (2012) National standards for diabetes self-management education and support. Diabetes Educ 38: 619-629.

5. Chodosh J, Morton SC, Mojica W, Maglione M, Suttorp MJ, et al. (2005) Meta-analysis: Chronic disease self-management programs for older adults. Ann Internal Medicine 143: 427-438.
6. Polonsky WH, Earles J, Smith S, Pease DJ, Macmillan M, et al. (2003) Integrating medical management with diabetes self-management training: A randomized control trial of the diabetes outpatient intensive treatment program. Diabetes care 26: 3048-3053.

7. Brown SA (1988) Effects of educational interventions in diabetes care: A meta-analysis of findings. Nur Res 37: 223-230.

8. Ismail K, Winkley K, Rabe-Hesketh S (2004) Systematic review and meta-analysis of randomised controlled trials of psychological interventions to improve glycaemic control in patients with type 2 diabetes. Lancet 363: 15891597.

9. Brown SA, Hanis CL (1999) Culturally competent diabetes education for mexican americans: The starr county study. Diabetes Educ 25: 226-236.

10. Two Feathers J, Kieffer EC, Palmisano G, Anderson M, Sinco B, et al. (2005) Racial and Ethnic Approaches to Community Health (REACH) Detroit partnership: Improving diabetes-related outcomes among African American and Latino adults. Am J Public Health 95: 1552-1560.

11. Coonrod BA, Betschart J, Harris MI (1994) Frequency and determinants of diabetes patient education among adults in the U.S. Population. Diabetes care 17: 852-858

12. Graziani C, Rosenthal MP, Diamond JJ (1993) Diabetes education program use and patient-perceived barriers to attendance. Fam med 31: 358-363.

13. Li R, Shrestha SS, Lipman R, Burrows NR, Kolb LE, et al. (2014) Diabetes self-management education and training among privately insured persons with newly diagnosed diabetes-United States, 2011-2012. Morbidity and Mortality Weekly Report (MMWR) 63: 1045-1049.

14. Walker EA, Silver LD, Chamany S, Schechter CB, Gonzalez JS, et al. (2014) Baseline characteristics and Latino versus non-Latino contrasts among Bronx A1C study participants. West J Nurs Res 36:1030-1051.

15. Siminerio LM (2006) Implementing diabetes self-management training programs: Breaking through the barriers in primary care. Endocr Pract 12: 124130.

16. Aubert RE, Herman WH, Waters J, Moore W, Sutton D, et al. (1998) Nurse case management to improve glycemic control in diabetic patients in a health maintenance organization. A randomized, controlled trial. Ann Intern Med 129: 605-612.

17. Glazier RH, Bajcar J, Kennie NR, Willson K (2006) A systematic review of interventions to improve diabetes care in socially disadvantaged populations. Diabetes Care 29: 1675-1688.

18. Siminerio LM, Piatt GA, Emerson S, Ruppert K, Saul M, et al. (2006) Deploying the chronic care model to implement and sustain diabetes self-management training programs. Diabetes Educ 32: 253-260.

19. Farrell P, Barnaby S, Galarza T, Simonson JK, Zonszein J, et al. (2013) Population management of diabetes in a high-need urban community in the Bronx: the experience of Montefiore Medical Center. Diabetes Educ 39: 515-522.

20. Knowler WC, Barrett-Connor E, Fowler SE, Hamman RF, Lachin JM, et al. (2002) Reduction in the incidence of type 2 diabetes with lifestyle intervention or metformin. N Engl J Med 346: 393-403.

21. Bozack A, Millstein S, Garcel JM, Kelly K, Ruberto R, et al. (2014) Implementation and Outcomes of the New York State YMCA Diabetes Prevention Program: A Multisite Community-Based Translation, 2010-2012. Prev Chronic Dis 11: 140006

22. Brown SA, Blozis SA, Kouzekanani K, Garcia AA, Winchell M, et al. (2005) Dosage effects of diabetes self-management education for Mexican Americans: the starr county border health initiative. Diabetes care 28: 527-532. 